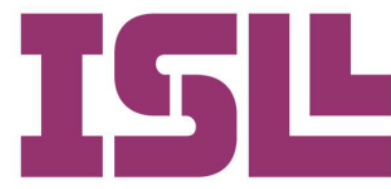

Recibido:

Noviembre 2013

Aprobado:

Enero 2014

Pág. 15 a la 23

Palabras clave
cuento musical,
competencia literaria,
discurso metaficcional,
intertextualidad.

\section{Keywords}

musical tale, literary skill, metafictional discourse, intertextuality.

\title{
Un nuevo enfoque de la lectura musical. Análisis literario, musical y didáctico del cuento Mi madre la Oca
}

\author{
María Isabel de Vicente-Yagüe Jara \\ Universidad de Murcia
}

\section{Resumen}

Nuestra experiencia docente en las aulas de diferentes niveles educativos nos alerta de la urgente necesidad de replantearnos nuevos enfoques de lectura. Consideramos que los esquemas narrativos tradicionales deben ser enriquecidos y actualizados por medio de diversos códigos que pretendan un motivador acercamiento al hecho literario, iniciativa ya tomada por ciertas colecciones de cuentos a través de una innovadora propuesta musical.

En este sentido, analizamos las cualidades didácticas e interartísticas del cuento musical Mi madre la Oca con texto de Fernando Palacios, música de Maurice Ravel e ilustraciones de Alicia Cañas. El diálogo que en el cuento se establece entre los distintos códigos (verbal, musical y gráfico), a partir de las relaciones intertextuales con la cuentística francesa fundamentalmente, da lugar a una triple lectura hipertextual y metaficcional, que aleja al escolar de un tradicional concepto lectoliterario mediante este atractivo formato editorial del libro-CD y procura el desarrollo de su competencia literaria.

\section{Abstract}

Our teaching experience in classrooms of different educational levels alert us of the urgent need to rethink new approaches to reading. We believe that traditional narrative schemes should be enriched and updated by various codes that seek a motivating approach to the literary fact, an initiative already taken by certain collections of stories through an innovative musical approach.

Hereby, in that sense, we analyze the didactic and interartistic qualities of the musical tale Mi madre la Oca written by Fernando Palacios, with music by Maurice Ravel and illustrations by Alicia Cañas. The dialogue in the story, developed between the various codes (verbal, musical and graphical) from the intertextual relations with the mainly French short stories, leads to a triple hypertextual and metafictional reading, which moves away from a traditional concept to school literary readers by publishing this attractive book-CD format, and tries to develop their literary skills. 


\section{Introducción}

Nuestra experiencia docente en las aulas de diferentes niveles educativos nos alerta de la urgente necesidad de replantearnos nuevos enfoques de lectura, atractivos diseños editoriales y, en definitiva, diferentes estrategias de motivación que procuren el fomento del hábito lector en el alumnado. En la actualidad, vivimos en una sociedad tecnológica, digital y mediática, repleta de estímulos que repercuten negativamente en el placer y disfrute de la actividad recogida e individual de lectura. Por ello, consideramos que los esquemas narrativos tradicionales deben ser enriquecidos o dicho de otro modo, actualizados por medio de nuevos códigos que pretendan distintos acercamientos al hecho literario, así como contextualizadas vivencias y experiencias lectoras que incluyan los libros en el quehacer cotidiano de los estudiantes.

En este sentido, a las estrategias metaficcionales de la postmodernidad representada en el género infantil del álbum ilustrado, habría que añadir la iniciativa de ciertas colecciones de cuentos a través de una innovadora propuesta musical. Más concretamente, analizamos en este trabajo las cualidades didácticas e interartísticas que exhibe el cuento musical titulado $\mathrm{Mi}$ madre la Oca con texto de Fernando Palacios, música de Maurice Ravel e ilustraciones de Alicia Cañas. El diálogo que en el cuento se establece entre los diversos códigos (verbal, musical y gráfico), a partir de las relaciones intertextuales con la cuentística francesa fundamentalmente, presenta como principal fin estimular y dirigir la imaginación infantil. La fantasía del escolar queda así expuesta a una triple lectura, un triple placer estético, que lo aleja de un tradicional concepto de lectura en este nuevo formato editorial del libro-CD.

\section{Mi madre la Oca: un texto entre textos}

Aludiendo al trabajo coordinado por Mendoza (2008), nuestro cuento objeto de análisis posee la cualidad literaria, artística, receptiva y didáctica de ser un texto entre textos. Mi madre la Oca representa ese "mosaico de citas" del que nos hablaba Kristeva (1969) y que pone de manifiesto el juego intertextual entre diversos textos, en este caso procedentes de la tradición literaria y musical. Maurice Ravel y la cuentística francesa fundamentalmente son los motores creativos del resultado hipertextual diseñado por Fernando Palacios, responsable artístico asimismo de la colección de cuentos musicales "La mota de polvo" en la que se encuadra el cuento que nos ocupa.

En primer lugar, el texto musical, principal hilo conductor del cuento Mi madre la Oca, recoge no solo la obra orquestal homónima del compositor francés Ravel, Ma mère l'oye, sino que además incluye, como marco de las cinco piezas que la componen, Pavana para una infanta difunta y el "Rigodón" de La tumba de Couperin de su misma autoría. El orden de los distintos números en el cuento musical, por tanto, es el siguiente:

Pavana para una infanta difunta

Ma mère l'oye

- Pavana de la Bella Durmiente 


$\begin{array}{ll}\circ & \text { Pulgarcito } \\ \circ & \text { Feuchilla, Emperatriz de las Pagodas } \\ \circ & \text { Las conversaciones de la Bella y la Bestia } \\ \circ & \text { El jardín encantado }\end{array}$

- $\quad$ La tumba de Couperin: Rigodón

En realidad, la versión original de Ma mère l'oye de Ravel fue compuesta para piano a cuatro manos entre septiembre de 1908 y abril de 1910 en París. Estas "cinco piezas infantiles" estaban dedicadas a los hijos de sus amigos Godebski, Jean y Marie, y de igual forma, fue estrenada por un dúo infantil de intérpretes, Geneviève Durony y Jeanne Leleu, el 20 de abril de 1910 en la Sala Gaveau de París, primer concierto de la Sociedad Musical Independiente. No obstante, la versión orquestal, escrita posteriormente en 1911, ha adquirido una mayor fama mundial; Tranchefort llega a comentar, incluso, que la versión orquestal consigue eclipsar a la pianística (1990, p. 620). Unos meses después, Ravel convertiría la suite de cinco piezas en ballet, añadiendo un preludio, un nuevo número ("Danza de la rueca") y ciertos interludios de unión entre las escenas; este ballet fue dedicado a Jacques Rouche y estrenado bajo la dirección de Gabriel Grovlez el 28 de enero de 1912 (Tranchefort, 2008, p. 936).

Desde el propio título de la obra, Ravel alude a la tradición cuentística francesa, representada en el personaje de Mamá Oca, mujer de campo, creadora de historias y poemas. Sin duda, fue Charles Perrault quien contribuyó a su difusión en el panorama de la literatura de tradición oral en su libro de relatos Histoires ou Contes du Temps passé, subtitulado Les Contes de ma Mère l'Oye, publicado en 1967. Mamá Oca era presentada como la autora de los cuentos que Perrault recopiló, autoría que ponía de manifiesto el carácter oral de los mismos. Así pues, de los cuentos del mencionado volumen, Ravel seleccionó como inspiradores de su obra musical "La Bella Durmiente del bosque" y "Pulgarcito".

Además de Perrault, Ravel dialoga a lo largo de su composición con los cuentos "Serpentino verde" de Madame Marie-Catherine d'Aulnoy y "La Bella y la Bestia" de Madame Leprince de Beaumont. Los cuentos de hadas del siglo XVII francés conforman, por tanto, el hipotexto del hipertexto musical final.

En consonancia con este mundo de fantasía e ingenuidad infantil, Ravel concibió cada una de las piezas de Ma mère l'oye. Subrayando las palabras del propio compositor francés, "La idea de evocar en estas piezas la poesía de la infancia me condujo naturalmente a simplificar mi manera de hacer, a despojar mi escritura" (Tranchefort, 1990, p. 620). Por otra parte, a modo de hilo programático, observamos en la misma partitura, al comienzo de algunas de las piezas, una cita textual del cuento homónimo. En "Petit Poucet", podemos leer, como se advierte en la figura 1, "Il croyait trouver aisément son chemin par le moyen de son pain qu'il avait semé partout où il avait passé; mais il fut bien surpris lorsqu'il n'en put 
retrouver une seule miette; les oiseaux étaient venus qui avaient tout mangé. (Ch. Perrault.)" (Ravel, 1912, p. 4).

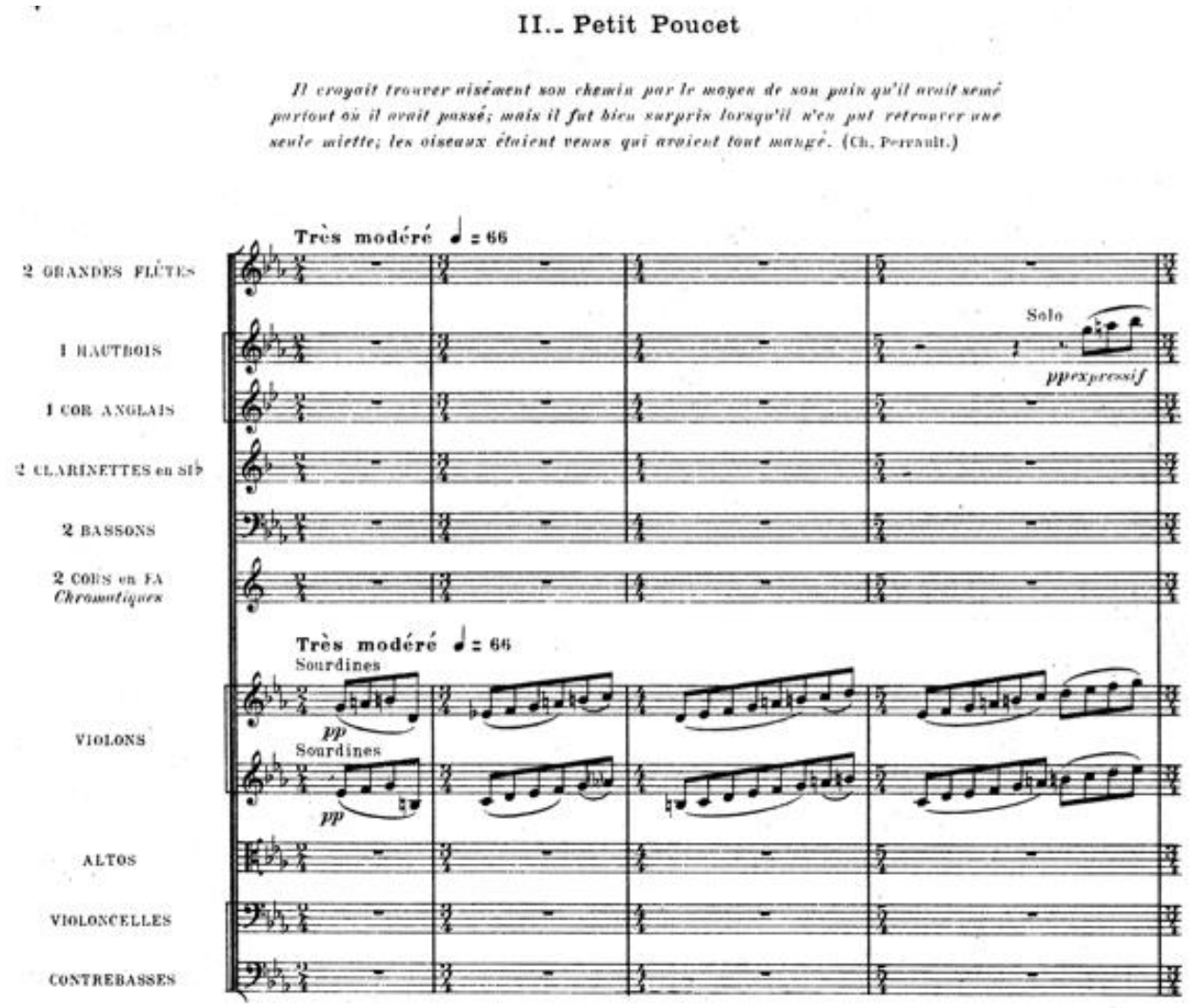

Figura 1. Ma mère l'oye: "Petit Poucet".

Fuente: Ravel (1912, p. 4)

Por su parte, Palacios, al igual que hiciera con la sección musical de su cuento, añadiendo dos números a los cinco de Ma mère l'oye de Ravel, no solo recurre en su discurso literario a los cuatro cuentos escogidos por Ravel para su musicalización, sino que también incluye un moderno y, por otra parte, intertextual y metaficcional relato de Jonathan Allen: ¡Despierta, Bella Durmiente! Palacios consigue armonizar en el texto inter-artístico que nos ocupa la ficción literaria de tradición oral y el hipotexto de Ravel (ya en sí mismo un hipertexto artístico de inspiración literaria), desde una innovadora perspectiva dirigida a la actualización y enriquecimiento del intertexto lector del joven público (Mendoza, 2001), al fomento del hábito lector y al desarrollo estético y creativo que la música, como lenguaje artístico, protencia.

Palacios parte de un escenario marco en el que sitúa la narración de los distintos relatos al auditorio infantil, pues el narrador del cuento musical se convierte en un cuentacuentos con unos oyentes explícitos, es decir, asume el papel del personaje Mamá Oca que da título al texto analizado. El cuento musical se inscribe, pues, en la tradición literaria de los cuentos de Sherezade de Las mil y una noches, el Decamerón de Bocaccio y los Cuentos de Canterbury de Chaucer, entre otros, retomando el valor social del cuento como relato oral. A continuación, podemos advertir cómo el narrador finaliza el quinto episodio: “Así es como 
el escritor francés Perrault escribió este cuento de La Bella Durmiente del bosque. Desde entonces se ha contado millones de veces: padres a hijos, abuelas a nietos, como os lo he contado yo a vosotros es como me lo contó mi abuela a mí" (Palacios, 2003, "Vuelve La bella durmiente del bosque" ${ }^{1}$.

Palacios adopta en su texto la figura del autor implícito que adelanta metanarrativamente aspectos del discurso, ofrece informaciones detalladas acerca de los personajes y aporta datos para la correcta interpretación de la historia. Este carácter metanarrativo se comprueba en los comentarios y reflexiones sobre la estructura y orden del discurso, que muestran las técnicas de construcción de la ficción narrativo-musical: "Y aquí llega mi segundo momento maravilloso del cuento: Pulgarcito, que es muy listo, deja una hilera de migas de pan para poder volver a casa. Las migas suenan así en el pizzicato de los contrabajos con sordina. א" (Palacios, 2003, "Pulgarcito").

El lector se convierte en un agente activo de la historia desde el momento en que el autor apela directamente a su persona para responder, fabular o completar la historia narrada a partir de su experiencia lectora previa, poniendo en su juego su conciencia intertextual. El tiempo del lector se incluye en el de la historia en el mismo instante en el que el narrador solicita la participación de su interlocutor literario:

Me inquietaba la melodía de la flauta mágica de El Flautista de Hamelin, que hipnotizaba a ratones y a niños. $\delta$ También me producía temblor escuchar aquello de ¡el país de irás y no volverás! $\delta$ Y ¿qué me decís cuando se abría la gruta de Alí Babá y los 40 ladrones al grito de “Ábrete Sésamo!!”? (Palacios, 2003, Introducción).

¿Cuál es?... exacto: La Bella durmiente del bosque. (Palacios, 2003, "La bella durmiente").

De igual forma, advertimos la alusión al poder narrativo de la música que realiza Palacios en el transcurso del cuento. La música, en calidad de arte del tiempo, es capaz de suscitar en el oyente a partir de estímulos sonoros el devenir de sucesos y experiencias ficticias y fantásticas. En este sentido, Palacios refiere de forma explícita la capacidad de contar, relatar y conversar de la música en ciertos ejemplos como los siguientes: "Escuchemos ahora cómo la orquesta es capaz de contarnos este segundo momento mágico del cuento de Pulgarcito" (Palacios, 2003, "Pulgarcito") y "Los instrumentos de cuerda también conversan. Observad cómo entran las violas, después los violines segundos y más tarde los violines primeros א" (Palacios, 2003, "Guía didáctica": "Preparación a la audición”). No obstante, al margen de las referencias y comparaciones concretas que el autor pueda destacar, el papel de la música a lo largo del relato supone un apoyo argumental significativo, que complementa desde una perspectiva constructiva el conjunto estético estudiado.

Con respecto al código plástico, tercer componente del objeto artístico plural, observamos en qué medida ofrece las claves contextuales de la historia, facilitando la lectura

\footnotetext{
${ }^{1}$ Debido a la inexistencia de numeración en las páginas del cuento musical analizado, de aquí en adelante citaremos el texto extraído del mismo mediante la referencia al título del capítulo en el que se ubica.
} 
literaria y procurando el reconocimiento visual de lo expresado por el código escrito. La información que aportan las ilustraciones posibilita al niño anticipar y atribuir significado a lo leído o escuchado a lo largo del proceso de recepción. Principalmente, las ilustraciones de Alicia Cañas (veamos un ejemplo en la figura 2) presentan al personaje del cuento aludido, lo que permite al lector una rápida identificación del mismo en su imaginario infantil, forjado a partir de su experiencia literaria previa, ya sea oral, escrita o visual.

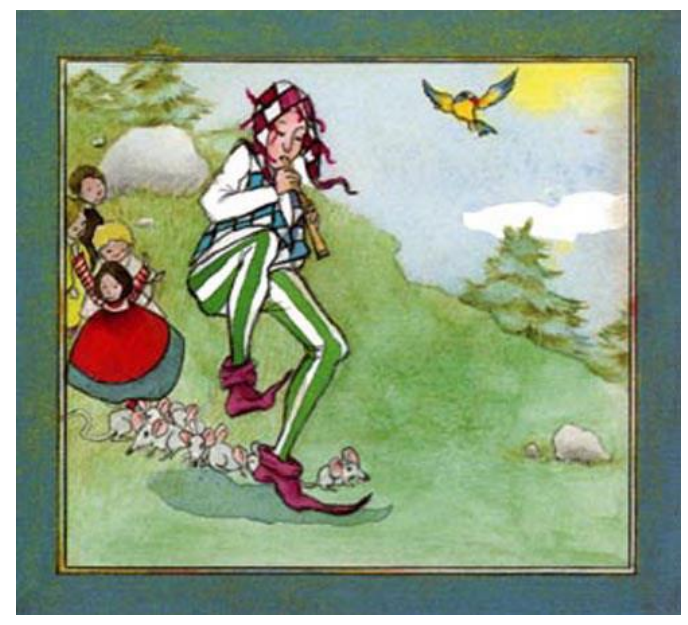

Figura 2. Ilustración de Alicia Cañas Cortázar.

Fuente: Mi madre la Oca (Palacios, 2003)

Por tanto, el elemento lúdico es fundamental en un tipo de cuento como el que respalda la colección "La mota de polvo", pues la interacción texto-música-imagen requiere una lectura alejada de los patrones tradicionales literarios, fundamentados en la lógica causal y arquetípica de los modelos preexistentes. Además, el propio cuento musical posibilita una doble vía de acceso, una doble vía receptiva de disfrute y "uso" del soporte literario-musical. Por una parte, disponemos de un libro con el cuento ilustrado de Mi madre la Oca, el cual admite ser leído de manera simultánea a la escucha del disco que narra el cuento acompañado de la música compuesta por Ravel (pistas 1-14). Por otra, en la segunda parte del disco (pistas 15-21), encontramos duplicada la sección musical, esta vez sin narrador, con el fin de que sea el mismo lector quien realice la función del cuentacuentos, acompasando música y texto según las indicaciones del cuento.

Finalmente, la estrategia utilizada por Palacios en su último episodio, "Los cuentos al revés", refleja una de las técnicas de invención de historias que ya proponía Gianni Rodari (2011, pp. 85-86) en su Gramática de la fantasía, con un capítulo de idéntico título. La imaginación, la creatividad y el disparate son los pilares sobre los que se sustenta esta ingeniosa y productiva manera de jugar con la literatura, re-crear lo ya creado y fomentar nuevas perspectivas de escritura y, por tanto, de lectura. La "inversión premeditada" (Rodari, 2011, p.85) de los temas procedentes de la cuentística tradicional son presentados bajo el signo de la postmodernidad, al entroncar literatura y actualidad en los siguientes términos: "Con la ayuda de este Rigodón de Ravel os contaré los últimos chismes de los cuentos famosos, una especie de 'revista del corazón de las hadas', ‘¡el cotilleo de los cuentos!'” 
(Palacios, 2003, "Los cuentos al revés"). Leamos algunos de estos "chismes": "El único animal que consiguió el título de aparejador fue el tercero de Los tres cerditos, aquel que supo hacerse una casa resistente utilizando productos de Ikea", "En realidad, Barbazul era un viejecito inofensivo que se teñía su barba blanca. Por eso sus esposas se suicidaban cuando se enteraban de que no era azul" (Palacios, 2003, "Los cuentos al revés").

\section{Conexión curricular competencial}

El cuento finaliza con una guía didáctica de lectura y audición, acentuando sus posibilidades de empleo en el aula a partir de las indicaciones contenidas en los siguientes apartados: "Sobre su contenido", "Quiénes han realizado este cuento", "Preparación a la audición". Si nos detenemos en el currículo de Educación Primaria (MEC, 2006), etapa en la que resultaría de un gran interés trabajar el cuento analizado, constatamos que la mayor parte de las competencias básicas son desarrolladas a través de su lectura-audición. En primer lugar, no cabe duda de que la competencia en comunicación lingüística es la principal en el conjunto establecido en el actual marco curricular de convergencia europea. El soporte textual y sus posibles vías de acceso, permitiendo no solo una lectura silenciosa sino también una lectura expresiva a modo de "karaoke musical", según sugiere el propio Palacios, procura en este último caso el desarrollo de las habilidades orales frecuentemente desatendidas en el panorama actual de la enseñanza de la lengua (Martín, 2009; Nuñez, 2008; Prado, 2004). Igualmente, el relato artístico hipertextual estudiado activa el intertexto lector del alumnado, a partir de la rica red de conexiones intertextuales que entreteje Mi madre la Oca, fomentando el hábito lector de los niños, motivados por el reconocimiento de personajes y aventuras procedentes de su incipiente experiencia literaria.

La competencia cultural y artística entra en juego en su triple vertiente musical, plástica y literaria. La adquisición de la misma por medio de la múltiple perspectiva receptivainterpretativa empuja y promueve la conciencia artística metaficcional del alumno, quien puede comprobar en las reflexiones y comentarios del propio autor a lo largo del cuento el proceso y las técnicas de composición literaria y musical ya comentados (estructura, intertextualidad, cuentos al revés...).

Las competencias transversales del tratamiento de la información y competencia digital, así como la competencia para aprender a aprender son abordadas explícitamente en la guía didáctica final del cuento, a través de las indicaciones teóricas, la manipulación digital de las pistas musicales y la dimensión metacognitiva del proceso de aprendizaje. En esta línea, el cuento insta a la reflexión sobre su misma estructura y significado, e igualmente invita a explorar y escuchar los elementos tímbricos en calidad de herramientas narrativas.

Relacionado con este último aspecto, destacamos el desarrollo de la competencia en el conocimiento y la interacción con el mundo físico, puntualizada básicamente en la exploración sensorial consciente de la música como fenómeno acústico. En no pocas ocasiones, se pretende la identificación tímbrica de los instrumentos con ciertos personajes en concreto: 
Está despierta y habla a ritmo de vals y con voz de clarinete. $\delta$ Y, junto a ella, un ser horrible, mezcla de león, oso y pantera, representado por el contrafagot. $\delta$ [...] El arpa y el violín hacen de varita mágica: el arpa, con su patinaje por las cuerdas; y el violín, tocando el mismo tema de la Bestia, pero muy agudo. $\delta$ Estas son Las conversaciones de la Bella y la Bestia (Palacios, 2003, "La bella y la bestia”).

La adquisición de la competencia social y ciudadana se promueve en su dimensión ética principalmente, ya que los relatos descubren una moraleja o enseñanza final con el fin de orientar las conductas de sus lectores en el mundo extraliterario. Por último, la autonomía e iniciativa personal cobra una especial relevancia en toda tarea que implica la lectura interpretativa de un texto literario o la escucha crítica de una composición musical. Y es aquí donde podemos resaltar la importancia del intertexto lector o artístico, mencionado anteriormente, dado que será ese bagaje o conocimiento cultural, literario y personal previo el que dirigirá la interpretación última del conjunto, potenciando la iniciativa, creatividad e imaginación del niño.

\section{A modo de colofón}

La intercomunicación en este cuento con formas ajenas al código escrito muestra un nuevo enfoque de lectura literaria, que persigue combatir la problemática creciente actual de la desmotivación lectora entre el joven público escolar, según se ha investigado recientemente (Guerrero, 2008; Vicente-Yagüe, 2012a, 2012b). El lenguaje musical pretende orientar, en este sentido, el texto literario de forma emotiva e igualmente constructiva y generadora de nuevos significados, pues la simbiosis de ambos códigos en el desciframiento del sentido global contempla una perspectiva distinta a la que proporciona la suma de cada uno de los códigos de forma aislada. El texto, la música, las ilustraciones, el formato, la tipografía actúan como un todo en el cuento musical de Mi madre la Oca. Se presenta necesaria una lectura que transcienda de lo meramente argumental y se oriente hacia el placer estético de este objeto artístico provocado por la conjunción de la palabra, la sensibilidad y emotividad musical y la imagen. Nos encontramos, por tanto, ante nuevas formas de lectura, dominadas por técnicas y estrategias metaficcionales y postmodernas (Amo, 2003; Colomer, 1998).

Finalmente, tras el análisis realizado en las líneas precedentes, consideramos que a través de este tipo de creaciones de signo hipertextual es posible reconducir la educación lectora de nuestros alumnos, con el fin de desarrollar su competencia literaria, en el marco de una educación integral articulada desde el enfoque competencial propuesto en los currículos vigentes.

\section{Bibliografía}

Amo, J.M. de (2003). Literatura infantil. Málaga: Aljibe.

Colomer, T. (1998). La formación del lector literario. Narrativa infantil y juvenil actual. Madrid: Fundación Germán Sánchez Ruipérez. 
Guerrero, P. (2008). Metodología de Investigación en Educación Literaria (El Modelo Ekfrástico). Murcia: Diego Marín.

Kristeva, J. (1969). Semeiotike. Recherches pour une sémanalyse. París: Seuil.

Martín, R.A. (2009). Manual de Didáctica de la Lengua y la Literatura. Madrid: Síntesis.

MEC (2006). Real Decreto 1513/2006, de 7 de diciembre, por el que se establecen las enseñanzas mínimas de la Educación Primaria (BOE, 8/12/2006).

Mendoza, A. (2001). El intertexto lector. El espacio de encuentro de las aportaciones del texto con las del lector. Cuenca: Universidad de Castilla-La Mancha.

Mendoza, A. (coord.) (2008). Textos entre textos. Las conexiones textuales en la formación del lector. Barcelona: Horsori Editorial.

Núñez, Ma P. (2008). Aspectos básicos de la didáctica de la lengua oral. En Jiménez, Ma A., Molina, Ma J., Rico, A.Mª, Ramos, A.M ${ }^{\mathrm{a}}$ y Rienda, J., Nociones didácticas sobre oralidad y escritura (pp. 35-55). Granada: Grupo Editorial Universitario.

Palacios, F. (dir.) (2003). Mi madre la Oca [CD-libro]. Col. La Mota de Polvo. VitoriaGasteiz: AgrupArte Producciones.

Prado, J. (2004). Didáctica de la lengua y la literatura para educar en el siglo XXI. Madrid: La Muralla.

Ravel, M. (1912). Ma mère l'oye. París: Durand \& Cie.

Rodari, G. (2011). Gramática de la fantasía. Introducción al arte de inventar historias. Barcelona: Planeta.

Tranchefort, F.-R. (dir.) (1990). Guía de la música de piano y de clavecín. Madrid: Taurus.

Tranchefort, F.-R. (dir.) (2008). Guía de la música sinfónica (2a ed.). Madrid: Alianza.

Vicente-Yagüe, M ${ }^{a}$ I. de (2012a). El Quijote en Manuel de Falla. Estrategias didácticas musicales para la enseñanza literaria en Educación Primaria. En Alonso, J.I., Escarbajal, A. y Nortes, R. (eds.), Experiencias de innovación en Educación Infantil y Educación Primaria (pp. 259-269). Murcia: Universidad de Murcia.

Vicente-Yagüe, M M $^{\mathrm{a}}$ I. de (2012b). Miguel Hernández y sus trovadores ideológicamente comprometidos. Una experiencia musical en la educación literaria. Lenguaje y textos. Revista de la Sociedad Española de Didáctica de la Lengua y la Literatura, 35, 77-83. 